\title{
THE HADAMARD THEOREM FOR PERMANENTS
}

\section{MARVIN MARCUS}

1. Statement of the result. Let $A=\left(a_{i j}\right)$ be an $n$-square non-negative hermitian matrix. A classical inequality of Hadamard states that

$$
\operatorname{det} A \leqq \prod_{i=1}^{n} a_{i i}
$$

with equality if and only if $A$ has a zero row (and column) or $A$ is a diagonal matrix: $A=\operatorname{diag}\left(a_{11}, \cdots, a_{n n}\right)$. Several generalizations are known (e.g. [1]).

Let per $A$ denote the permanent of $A$,

$$
\operatorname{per} A=\sum_{\sigma} \prod_{i=1}^{n} a_{i \sigma(i)},
$$

where the summation extends over the whole symmetric group of degree $n$. It was conjectured in [4] that in analogy with (1),

$$
\operatorname{per} A \geqq \prod_{i=1}^{n} a_{i i}
$$

with the conditions of equality precisely those in the Hadamard determinant theorem. L. Mirsky recently listed this conjecture among several other problems concerning the permanent function [5]. This conjecture was suggested by an inequality of I. Schur [6] (see also [4]):

$$
\text { per } A \geqq \operatorname{det} A \text {. }
$$

In an unsuccessful attempt [3] to prove (3) H. Minc and the present author obtained an inequality of the form

$$
\text { per } A \geqq c_{n} \prod_{i=1}^{n} a_{i i}
$$

in which the constant $c_{n}$ depends only on $n$ and not on $A$.

It is the purpose of this paper to present the proof of an inequality that substantially generalizes (3) and to discuss the somewhat delicate cases of equality. Let $A(i)$ denote the principal submatrix of $A$ obtained by deleting row and column $i$ of $A$. The main result is contained in the following

Received by the editors June 26, 1963. 
ThEOREM 1. Let $A=\left(a_{i j}\right)$ be an $(r+1)$-square non-negative hermitian matrix. Then

$$
(r+1) a_{i i} \text { per } A(i) \geqq \operatorname{per} A \geqq a_{i i} \operatorname{per} A(i), \quad 1 \leqq i \leqq n .
$$

If $A$ has a zero row then (4) is equality throughout. If $A$ has no zero row then the lower equality holds if and only if $a_{i i}$ is the only nonzero entry in row and column i of $A$; the upper equality holds if and only if the rank of $A$ is 1 .

The permanent is unaltered by pre- and post-multiplication by permutation matrices so that we can take $i=1$ in proving (4).

Once (4) is established it is clear that an obvious induction on $r$ will yield

THEOREM 2. If $A=\left(a_{i j}\right)$ is an $n$-square non-negative hermitian matrix then

$$
\text { per } A \geqq \prod_{i=1}^{n} a_{i i}
$$

with equality if and only if $A$ has a zero row or $A$ is a diagonal matrix.

2. Preliminaries. Let $U$ be an $n$-dimensional unitary space with inner product $(x, y)$. For $1 \leqq r \leqq n$ define $U^{(r)}$ to be the space of $r$ tensors on $U\left[2\right.$, Chapter 7]; that is, $U^{(r)}$ is the dual space of the space $M_{r}(U)$ of all multilinear functionals of $r$-tuples of vectors from $U$. If $x_{1}, \cdots, x_{r}$ are in $U$ then their tensor product $x_{1} \otimes \cdots \otimes x_{r} \in U^{(r)}$ is defined by

$$
x_{1} \otimes \cdots \otimes x_{r}(\phi)=\phi\left(x_{1}, \cdots, x_{r}\right), \quad \phi \in M_{r}(U) .
$$

An inner product in $U^{(r)}$ is given by

$$
\left(x_{1} \otimes \cdots \otimes x_{r}, y_{1} \otimes \cdots \otimes y_{r}\right)=\prod_{i=1}^{r}\left(x_{i}, y_{i}\right) .
$$

Define the completely symmetric operator $S^{(r)}: U^{(r)} \rightarrow U^{(r)}$ by

$$
S^{(r)}\left(x_{1} \otimes \cdots \otimes x_{r}\right)=\frac{1}{r !} \sum_{\sigma \in S_{r}} x_{\sigma(1)} \otimes \cdots \otimes x_{\sigma(r)},
$$

where $S_{r}$ is the symmetric group of degree $r$. The symmetric product of $x_{1}, \cdots, x_{r}$ is then defined by

$$
x_{1} \cdots x_{r}=S^{(r)}\left(x_{1} \otimes \cdots \otimes x_{r}\right) .
$$

The range space of $S^{(r)}$ is the symmetry class of completely symmetric 
tensors on $U$ denoted by $U_{(r)}$ and since it is a subspace of $U^{(r)}$ it is unitary. By combining (6), (7), (8) we compute that the inner product of two symmetric products is

$$
\left(x_{1} \cdots x_{r}, y_{1} \cdots y_{r}\right)_{r}=\frac{1}{r !} \operatorname{per}\left(\left(x_{i}, y_{j}\right)\right), \quad 1 \leqq i, j \leqq r .
$$

(The formula (9) is an immediate consequence of the fact that $S^{(r)}$ is idempotent and hermitian.) Next let $G_{r, n}$ denote the totality of nondecreasing sequences of length $r$ chosen from $1, \cdots, n$. If $\omega \in G_{r, n}$ let $\mu(\omega)$ be the product of the factorials of the multiplicities of the distinct integers in $\omega$; e.g. $\mu(3,3,7,7,7,9)=2 ! 3$ !. If $e_{1}, \cdots, e_{n}$ is an orthonormal (o.n.) basis of $U$ then the

$$
\left(\begin{array}{c}
n+r-1 \\
r
\end{array}\right)
$$

symmetric products $\sqrt{ }(r ! / \mu(\omega)) e_{\omega_{1}} \cdots e_{\omega_{r}},\left(\omega_{1}, \cdots, \omega_{r}\right)=\omega \in G_{r, n}$, constitute an o.n. basis of $U_{(r)}$ in the inner product $(,)_{r}$. We let $e_{\omega}=e_{\omega_{1}} \cdots e_{\omega_{r}}$.

3. Proofs. Assume that $A$ is $(r+1)$-square and has no zero row and let $D=\operatorname{diag}\left(a_{11}^{-1 / 2}, 1, \cdots, 1\right)$. Then the 1,1 entry of $B=D A D$ is 1 , per $B=a_{11}^{-1}$ per $A, A(1)=B(1)$, and $B$ is also non-negative hermitian. If we prove Theorem 1 for $B$ we will clearly have the result we want for $A$. Since $B$ is non-negative hermitian it follows that $B$ is a Gram matrix based on some set $e_{1}, v_{1}, \cdots, v_{r}$ where $e_{1}$ is a unit vector:

$$
\begin{aligned}
b_{11} & =1=\left(e_{1}, e_{1}\right), \\
b_{1, j+1} & =b_{j+1,1}=\left(e_{1}, v_{j}\right), \quad j=1, \cdots, r, \\
b_{i+1, j+1} & =\left(v_{i}, v_{j}\right), \quad i, j=1, \cdots, r .
\end{aligned}
$$

Let $e_{2}, \cdots, e_{n}$ be a completion of $e_{1}$ to an o.n. basis of $U$. Define a map $T: U_{(r)} \rightarrow U_{(r+1)}$ by $T\left(e_{\omega}\right)=e_{1} \cdot e_{\omega_{1}} \cdots e_{\omega_{r}}$, all $\omega=\left(\omega_{1}, \cdots, \omega_{r}\right)$ $\in G_{r, n}$, and extend linearly. It is an easy consequence of the symmetry and linearity of the symmetric product in its factors that

$$
T\left(x_{1} \cdots x_{r}\right)=e_{1} \cdot x_{1} \cdots x_{r}
$$

for all $x_{1}, \cdots, x_{r}$ in $U$. Let $R \subset U_{(r+1)}$ denote the range of $T$ and let $T^{*}$ denote the conjugate dual map of $T ; T^{*}: R \rightarrow U_{(r)}$. That is, $T^{*}$ satisfies

$$
(T h, g)_{r+1}=\left(h, T^{*} g\right)_{r} \quad \text { for all } h \in U_{(r)}, g \in R \text {. }
$$


Next let $f\left(x_{1}, \cdots, x_{r}\right)$ denote the Rayleigh quotient

$$
\begin{aligned}
f\left(x_{1}, \cdots, x_{r}\right) & =\frac{\left(T x_{1} \cdots x_{r}, T x_{1} \cdots x_{r}\right)_{r+1}}{\left(x_{1} \cdots x_{r}, x_{1} \cdots x_{r}\right)_{r}} \\
& =\frac{\left(T^{*} T x_{1} \cdots x_{r}, x_{1} \cdots x_{r}\right)_{r}}{\left(x_{1} \cdots x_{r}, x_{1} \cdots x_{r}\right)_{r}} .
\end{aligned}
$$

Now let $H$ denote the non-negative hermitian transformation $T^{*} T: U_{(r)} \rightarrow U_{(r)}$. It is easy to verify $[4$, Theorem 3$]$ that $x_{1} \cdots x_{r}=0$ if and only if some $x_{i}=0$. Thus $f\left(x_{1}, \cdots, x_{r}\right)$ is defined for all sets of $r$ nonzero vectors $x_{1}, \cdots, x_{r}$ in $U$ and it is known that such values of $f$ lie in the interval between the largest and smallest eigenvalues of $H$. We compute the eigenvalues of $H$ by obtaining a matrix representation of $H$. The basis $\sqrt{ }(r ! / \mu(\omega)) e_{\omega}, \omega \in G_{r, n}$, ordered lexicographically in $\omega$, is o.n. for $U_{(r)}$ and the $(\tau, \omega)$ entry of the matrix representation of $H$ in this ordered basis is

$$
\frac{r !}{\sqrt{ }(\mu(\omega) \mu(\tau))}\left(H e_{\omega}, e_{\tau}\right)_{r .}
$$

Now

$$
\begin{aligned}
\left(H e_{\omega}, e_{\tau}\right)_{r} & =\left(T^{*} T e_{\omega}, e_{r}\right)_{r}=\left(T e_{\omega}, T e_{\tau}\right)_{r+1} \\
& =\left(e_{1} \cdot e_{\omega_{1}} \cdots e_{\omega_{r}}, e_{1} \cdot e_{\tau_{1}} \cdots e_{r_{r}}\right) \\
& =\left(e_{(1, \omega)}, e_{(1, \tau)}\right)_{r+1}
\end{aligned}
$$

where $(1, \omega)$ is the sequence $\left(1, \omega_{1}, \cdots, \omega_{r}\right) \in G_{r+1, n}$ and similarly for $(1, \tau)$. The vectors $\sqrt{ }((r+1) ! / \mu(\alpha)) e_{\alpha}, \alpha \in G_{r+1, n}$, are an o.n. basis for $U_{(r+1)}$ in the inner product $(,)_{r+1}$. Moreover $(1, \omega)=(1, \tau)$ if and only if $\omega=\tau$. Hence from (12) we have

$$
\left(H e_{\omega}, e_{\tau}\right)_{r}=\frac{\mu((1, \omega))}{(r+1) !} \delta_{\omega, \tau} .
$$

Thus the matrix representation of $H$ in the basis $\sqrt{ }(r ! / \mu(\omega)) e_{\omega}$ is diagonal and the eigenvalues of $H$ are seen from (11) to be

$$
\begin{aligned}
\lambda_{\omega}(H) & =\frac{r !}{\mu(\omega)}\left(H e_{\omega}, e_{\omega}\right)_{r} \\
& =\frac{r !}{\mu(\omega)} \frac{\mu((1, \omega))}{(r+1) !} \\
& =\frac{1}{r+1} \frac{\mu((1, \omega))}{\mu(\omega)} .
\end{aligned}
$$


Clearly $\mu((1, \omega)) \geqq \mu(\omega)$ with equality if and only if $\omega_{1}>1$. Thus the minimum eigenvalue of $H$ is $1 /(r+1)$ and the symmetric tensors $e_{\omega}, \omega_{1}>1$, constitute the totality of corresponding eigenvectors. Suppose the multiplicities greater than 1 of the distinct integers in $\omega$ are $m_{1}, \cdots, m_{p}$. Then the multiplicities for $(1, \omega)$ are either $m_{1}, \cdots, m_{p}$ or $m_{1}+1, m_{2}, \cdots, m_{p}$. In the first instance $\omega_{1}>1$ and $\mu((1, \omega)) / \mu(\omega)$ $=1$; in the second $\omega_{1}=1$ and $\mu((1, \omega)) / \mu(\omega)=m_{1}+1$. This latter expression is maximal only when $m_{1}=r$, i.e., for the sequence $\omega=(1, \cdots, 1)$. Thus we conclude that

$$
1 \geqq f\left(x_{1}, \cdots, x_{r}\right) \geqq \frac{1}{r+1} .
$$

The lower equality holds if and only if $x_{1} \cdots x_{r}$ lies in the space spanned by the tensors $e_{\omega}, \omega_{1}>1, \omega \in G_{r, n}$. The upper equality holds if and only if $x_{1} \cdots x_{r}$ is a multiple of $e_{1} \cdots e_{1}$. Now by (10) we have

$$
\begin{aligned}
f\left(v_{1}, \cdots, v_{r}\right) & =\frac{\left(T v_{1} \cdots v_{r}, T v_{1} \cdots v_{r}\right)_{r+1}}{\left(v_{1} \cdots v_{r}, v_{1} \cdots v_{r}\right)_{r}} \\
& =\frac{\left(e_{1} \cdot v_{1} \cdots v_{r}, e_{1} \cdot v_{1} \cdots v_{r}\right)_{r+1}}{\left(v_{1} \cdots v_{r}, v_{1} \cdots v_{r}\right)_{r}} \\
& =\frac{\frac{1}{(r+1) !} \operatorname{per} B}{\frac{1}{r !} \operatorname{per} B(1)} \\
& =\frac{1}{r+1} \frac{\operatorname{per} B}{\operatorname{per} B(1)}
\end{aligned}
$$

and it follows from (13) that

$$
(r+1) \operatorname{per} B(1) \geqq \operatorname{per} B \geqq \operatorname{per} B(1) .
$$

As we indicated earlier (4) follows from (14).

We noted following (13) that the lower equality can hold if and only if

$$
v_{1} \cdots v_{r}=\sum c_{\omega} e_{\omega}
$$

where the summation extends over all $\omega \in G_{r, n}$ for which $\omega_{1}>1$. We prove that (15) implies that $\left(v_{i}, e_{1}\right)=0, i=1, \cdots, r$. Let $h$ denote the tensor on the right side of (15). Then 


$$
\left(v_{1} \cdots v_{r}, e_{1} \cdots e_{1}\right)_{r}=\prod_{k=1}^{r}\left(v_{k}, e_{1}\right)
$$

and

$$
\left(h, e_{1} \cdots e_{1}\right)_{r}=\sum_{\omega_{1}>1} c_{\omega}\left(e_{\omega}, e_{1} \cdots e_{1}\right)_{r}=0
$$

Hence some $\left(v_{j}, e_{1}\right)=0$ and from the symmetry of the symmetric product we can assume $j=1$. Suppose we have proved that $\left(v_{1}, e_{1}\right)$ $=\cdots=\left(v_{k}, e_{1}\right)=0$. Since no $v_{i}=0, i=1, \cdots, r$, we know $[4$, Theorem 3] that $0 \neq v_{1} \cdots v_{k} \in U_{(k)}$. The tensors $e_{\alpha}, \alpha \in G_{k, n}$, constitute a basis for $U_{(k)}$ and because $v_{1} \cdots v_{k} \neq 0$ there exists an $\alpha \in G_{k, n}$ for which $\left(v_{1} \cdots v_{k}, e_{\alpha}\right)_{k} \neq 0$. Let $\beta=\left(1, \cdots, 1, \alpha_{1}, \cdots, \alpha_{k}\right)$ $\in G_{r, n}$ where the notation means that $\alpha$ has been preceded with $r-k 1$ 's to make a nondecreasing sequence of length $r$. Now once again $\left(h, e_{\beta}\right)=0$ and hence

$$
\begin{aligned}
0 & =\left(v_{1} \cdots v_{r}, e_{\beta}\right)_{r}=\left(v_{1} \cdots v_{k} \cdot v_{k+1} \cdots v_{r}, e_{1} \cdots e_{1} \cdot e_{\alpha_{1}} \cdots e_{\alpha_{k}}\right)_{r} \\
& =\frac{1}{r !} \operatorname{per}\left(\begin{array}{c:c}
\left(v_{1}, e_{1}\right) \cdots\left(v_{1}, e_{1}\right) & \left(v_{1}, e_{\alpha_{1}}\right) \cdots\left(v_{1}, e_{\alpha_{k}}\right) \\
\vdots & \vdots \\
\left(v_{k}, e_{1}\right) \cdots\left(v_{k}, e_{1}\right) & \left(v_{k}, e_{\alpha_{1}}\right) \cdots\left(v_{k}, e_{\alpha_{k}}\right) \\
\hdashline\left(v_{k+1}, e_{1}\right) \cdots\left(v_{k+1}, e_{1}\right) & \left(v_{k+1}, e_{\alpha_{1}}\right) \cdots\left(v_{k+1}, e_{\alpha_{k}}\right) \\
\vdots & \vdots \\
\left(v_{r}, e_{1}\right) \cdots\left(v_{r}, e_{1}\right) & \left(v_{r}, e_{\alpha_{1}}\right) \cdots\left(v_{r}, e_{\alpha_{k}}\right)
\end{array}\right) .
\end{aligned}
$$

The upper left block in this last matrix is $k \times(r-k)$, the upper right block is $k \times k$, the lower left is $(r-k) \times(r-k)$ and the lower right is $(r-k) \times k$. The upper left block consists of zeros and hence by the Laplace expansion theorem for permanents we have

$$
\begin{aligned}
0 & =\operatorname{per}\left(\begin{array}{c}
\left(v_{1}, e_{\alpha_{1}}\right) \cdots\left(v_{1}, e_{\alpha_{k}}\right) \\
\vdots \\
\left(v_{k}, e_{\alpha_{1}}\right) \cdots\left(v_{k}, e_{\alpha_{k}}\right)
\end{array}\right) \operatorname{per}\left(\begin{array}{cc}
\left(v_{k+1}, e_{1}\right) \cdots\left(v_{k+1}, e_{1}\right) \\
\vdots \\
\left(v_{r}, e_{1}\right) \cdots\left(v_{r}, e_{1}\right)
\end{array}\right) \\
& =k !\left(v_{1} \cdots v_{k}, e_{\alpha}\right)_{k}(r-k) ! \prod_{j=k+1}^{r}\left(v_{j}, e_{1}\right) .
\end{aligned}
$$

Now $\left(v_{1} \cdots v_{k}, e_{\alpha}\right)_{k} \neq 0$ and hence some $\left(v_{j}, e_{1}\right)=0, j=k+1, \cdots, r$. We can assume $\left(v_{k+1}, e_{1}\right)=0$. Thus we have proved $\left(v_{i}, e_{1}\right)=0$, $i=1, \cdots, r$. In terms of the matrix $B$ this implies that $b_{1 j}=\bar{b}_{j 1}=0$, $j=2, \cdots, r+1$, and hence $B=1+B(1)$. 
If the upper equality holds then $v_{1} \cdots v_{r}$ was seen to be a multiple of $e_{1} \cdots e_{1}$. Since we are assuming that $A$ has no zero row it follows from [4, Theorem 3] that $v_{i}=d_{i} e_{1}, i=1, \cdots, r$, and hence $B$ is the Gram matrix based on the set $e_{1}, d_{1} e_{1}, \cdots, d_{r} e_{1}$. This means that $B$ and hence $A$ has rank 1 , say $A=\left(z_{i} \bar{z}_{j}\right), i, j=1, \cdots, r+1$. Of course, when $A$ has this form then

$$
\operatorname{per} A=(r+1) ! \prod_{j=1}^{r+1}\left|z_{j}\right|^{2}, \quad \operatorname{per} A(1)=r ! \prod_{j=2}^{r+1}\left|z_{j}\right|^{2}
$$

and clearly per $A=(r+1) a_{11}$ per $A(1)$. This completes the proof of Theorem 1.

\section{REFERENCES}

1. E. Fischer, Über den Hadamardschen Determinantensatz, Arch. Math. Phys. (3) 13 (1908) 32-40.

2. N. Jacobson, Lectures in abstract algebra, Vol. 2, Van Nostrand, New York, 1953.

3. M. Marcus and H. Minc, The Pythagorean theorem in certain symmetry classes of tensors, Trans. Amer. Math. Soc. 104 (1962), 510-515.

4. M. Marcus and M. Newman, Inequalities for the permanent function, Ann. of Math. (2) 75 (1962), 47-62.

5. L. Mirsky, Results and problems in the theory of doubly-stochastic matrices, Z. Wahrscheinlichkeitstheorie 1 (1963), 319-334.

6. I. Schur, Über endliche Gruppen und Hermitesche Formen, Math. Z. 1 (1918), 184-207.

University of California, Santa Barbara 